\title{
Simulation de la dégradation de la concentration d'un polluant en écoulement permanent bidimensionnel par la méthode des éléments finis.
}

\section{Simulation of a pollutant's concentration degradation in permanent two-dimensional flow using the finite element method.}

\author{
Deo Mwelwa' ${ }^{1}$ David llunga ${ }^{2}$, Jean-Paul Katond Mbay ${ }^{3}$ \\ ${ }^{1}$ Département d'Electromécanique, Faculté Polytechnique, Université de Lubumbashi, République Démocratique du \\ Congo, mwelwa.wamwelwa@gmail.com \\ ${ }^{2}$ Département d'Electromécanique, Faculté Polytechnique, Université de Lubumbashi, République Démocratique du \\ Congo,daoudika@yahoo.fr \\ ${ }^{3}$ Département d'Electromécanique, Faculté Polytechnique, Université de Lubumbashi, République Démocratique du \\ Congo, jpkatond@hotmail.com
}

RÉSUMÉ. Dans cette étude, nous effectuons une simulation de la dégradation d'un polluant en écoulement bidimensionnel par la méthode des éléments finis dans un domaine de 15 unités sur 15 de longueur. Pour y arriver, nous commençons par établir la formulation forte du comportement, ensuite nous la transformons en formulation variationnelle. Après avoir défini les données liées au domaine d'observation, nous fixons les conditions initiales et finales. Ce qui nous permet de résoudre le problème. Les résultats obtenus montrent que l'étendue de la zone impactée est directement proportionnelle à la vitesse de propagation et au coefficient de diffusion. Le niveau de concentration du polluant n'affecte que très minimalement cette étendu.

ABSTRACT. In this study, we do perform a simulation of a pollutant's degradation in two-dimensional flow using the finite elements method in an area of 15 units by 15 in length. To this end, we start by establishing the strong formulation of its behavior, then transform it to a variational formulation. After defining the data related to the observation domain, we set the initial and final conditions. This allows us to solve the problem. The results obtained show that the size of the impacted area is directly proportional to the speed of propagation and the diffusion coefficient. The pollutant's concentration level only very minimally affects this size.

MOTS-CLÉS. Simulation, Dégradation, Concentration, Polluant, Ecoulement, Eléments finis.

KEYWORDS. Simulation, Degradation, Concentration, Pollutant, Flow, Finite elements.

\section{Introduction}

La croissance économique d'un pays basée sur les mines passe aussi par la prolifération des industries. Certes ces industries dans leur objectif premier sont au service de l'homme en fabriquant des substances les plus diverses répondant aux besoins de la société ou d'ingénierie. Elles sont très souvent perçues comme des activités à risques qui résultent d'une part de l'augmentation constante de la puissance des unités de production, d'autre part de la nature combustible, explosive ou toxique des produits mis en œuvre ou fabriqués dans l'usine. Cette dernière nature toxique peut être causée par des émanations dans l'environnement des gaz nuisibles à la santé de l'homme. C'est ainsi que dans cette étude, nous nous proposons de simuler la dégradation d'un polluant en écoulement bidimensionnel par la méthode des éléments finis.

Les objectifs poursuivis dans cette étude sont :

- De déterminer les zones impactées et le niveau de concentration en polluant ;

- Et de montrer l'influence du flux de sortie de la source sur la zone impactée par le polluant.

- Pour y arriver, nous supposerons que : 
- Les pertes par dépôt sont négligées ;

- Le polluant n'est pas radioactif.

\section{Modélisation du comportement}

Comme montré à la figure II.1 ci-dessous, l'écoulement atmosphérique des polluants peut être modélisé par un comportement combiné d'advection et de diffusion.

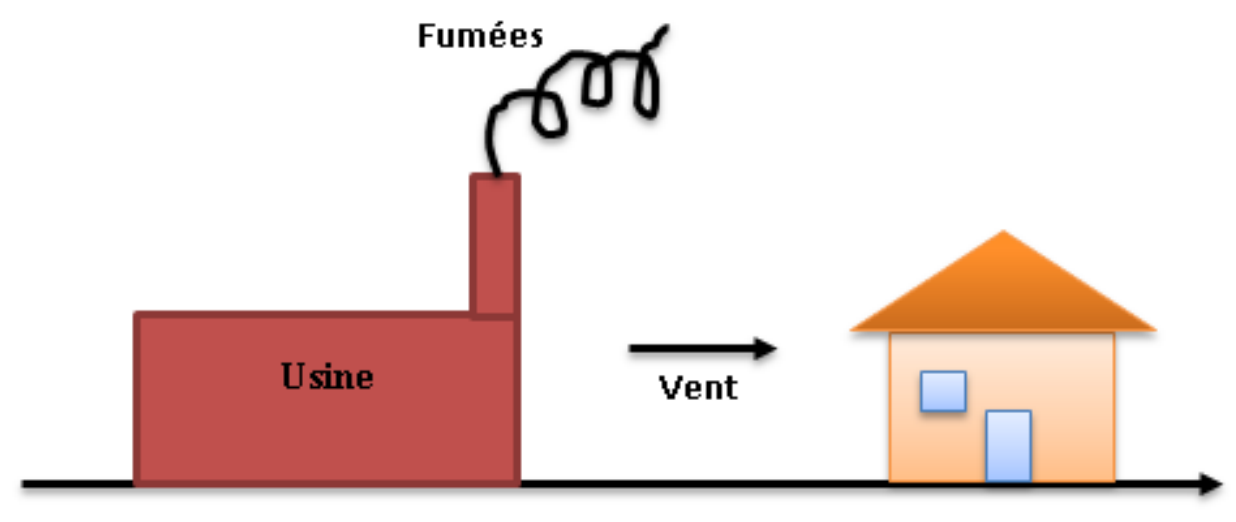

Figure II.1. Advection des fumées par le vent.

Les phénomènes de transport de masse sont faciles à observer dans notre environnement quotidien. Le mouvement du vent, la propagation d'un polluant dans l'océan ou l'air, et la lente dispersion de l'odeur d'un parfum sont tous des exemples de déplacement de masses d'un endroit à un autre. Deux phénomènes de transport de masse sont d'intérêts particuliers : la diffusion et l'advection [1].

Le mécanisme de diffusion a deux propriétés principales : Il est aléatoire dans la nature, et le transport va des régions de forte concentration à celles de faible concentration. Au niveau macroscopique, la diffusion se manifeste souvent comme un spectre observable de l'intensité, ou d'un gradient de concentration (voir la figure II.2). En outre, les points précédents ont défini l'advection comme le transport non aléatoire associé à l'écoulement du fluide. La combinaison de l'advection et de la diffusion est importante dans plusieurs applications de la dynamique des fluides [3] :

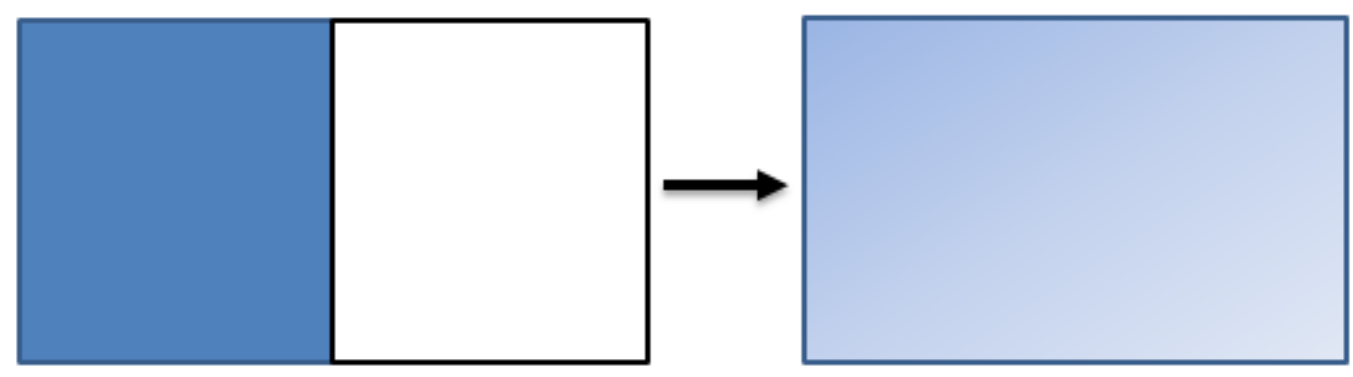

Figure II.2. Formation d'un gradient de concertation 
Son équation est déterminée de la manière suivante :

- La concentration C d'une substance dans un fluide

La quantité d'intérêt fondamental en matière d'environnement en mécanique des fluides est la concentration. Dans l'usage commun, le terme concentration exprime une mesure de la quantité d'une substance dans un fluide.

Mathématiquement, la concentration $C$ est le rapport de la masse $M$ d'une substance au volume du fluide [4] :

$$
C=\frac{\text { Masse de la substance }}{\text { volume du fluide }}=\frac{M}{v}\left[\mathrm{mg} / \mathrm{m}^{3}\right]
$$

\section{- Leflux}

Il n'est pas suffisant d'avoir la concentration dans un endroit, on a besoin de définir la quantité de substances qui se déplacent d'un endroit à un autre. On définit alors le flux [4].

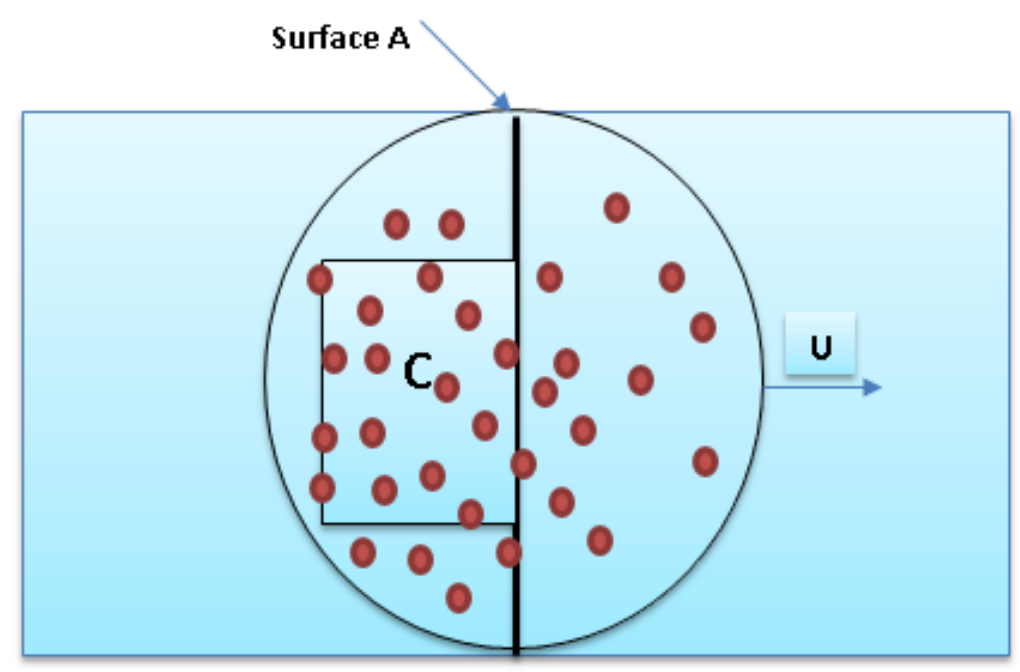

Figure II.3. Schéma d'un flux

En physique, le flux d'une substance dans une direction donnée est défini comme la quantité passante au travers d'une section perpendiculaire à cette direction par unité de surface et par unité de temps [3].

$$
\text { Flux }=q=\frac{\text { substance qui passe à travers la section }}{\text { section } * \text { durée de temps } \Delta t}=\frac{M}{A . \Delta t}
$$

\section{- Le flux d'advection}

Le flux d'advection est issu du mouvement du fluide porteur. Ce qui veut dire que :

$$
\begin{gathered}
q=\frac{\text { substance }}{\text { volume de fluide }} \frac{\text { volume de fluide }}{\text { surface.temps }}=\frac{M}{V} \cdot \frac{V}{A \cdot \Delta t}=\frac{C \cdot V}{V} \cdot \frac{V}{A \cdot \Delta t}=C \cdot \frac{V}{A \cdot \Delta t}=C \cdot \frac{A \cdot l}{A . \Delta t} \\
q=C . U
\end{gathered}
$$

Avec $U$, la vitesse d'entrainement du fluide porteur.

L'expression (II.3) signifie que le processus d'advection se fait par le transport passif de la substance d'un endroit à un autre à l'aide du fluide porteur en mouvement. 


\section{- L'équation d'advection pure}

Dans l'advection pure, on s'intéresse seulement au flux d'advection, et on néglige les autres flux comme par exemple le flux diffusif.

Soit à écrire le bilan de flux à travers la section $A$ durant le temps $d t$ dans un volume élémentaire :

$$
V=\Delta x . A \quad(I I .4)
$$

La variation de la masse durant un temps $d t$ correspond à la somme du flux interne (source dans V) et du flux externe (entrée et sortie) [3] :

$$
\begin{gathered}
{\left[\begin{array}{c}
\text { la variation de la masse } \\
\text { dans } V \text { durant } d t
\end{array}\right]=\left[\begin{array}{c}
\text { flux entrant } \\
- \\
\text { flux sortant }
\end{array}\right]+\left[\begin{array}{l}
\text { source } \\
\text { dans } V
\end{array}\right]} \\
{\left[\frac{d m}{d t}\right]=\left[\begin{array}{c}
\text { flux entrant } \\
- \\
\text { flux sortant }
\end{array}\right]+\left[\begin{array}{c}
\text { Source } \\
\text { dans } V
\end{array}\right]}
\end{gathered}
$$

Or, la variation de la concentration est donnée par :

$$
d C=\frac{d m}{v} \Rightarrow d m=v \cdot d c
$$

A $t$ fixé, le flux entrant par la section $A$ est $q(x, t) . A$ et le flux sortant par la section $A$ est $q((x+d x), t) \cdot A$, d'où la variation de la masse $d m$ dans un volume $V$ durant un temps $d t$ est comme suit :

$$
\frac{d m}{d t}=\frac{V \cdot d C}{d t}
$$

En remplaçant $I I .4$ dans $I I .6$, on obtient :

$$
\begin{aligned}
& \frac{A \cdot \Delta x \cdot d C}{d t}=[q(x, t) \cdot A]-[q(x+\Delta x, t) \cdot A] \\
& \Rightarrow \frac{d C}{d t}=-\frac{q(x+\Delta x, t)-q(x, t)}{\Delta x}
\end{aligned}
$$

En tenant compte du flux interne c'est-à-dire de la source $r$, le bilan devient :

$$
\begin{array}{r}
\frac{d C}{d t}=-\frac{q(x+\Delta x, t)-q(x, t)}{\Delta x}+r=-\frac{\partial q}{\partial x}+r \\
\Rightarrow \quad \frac{\partial C}{\partial t}=-U \frac{\partial C}{\partial x}+r \quad \text { (II.11) }
\end{array}
$$

En extrapolant l'étude en $2 \mathrm{D}$ et pour une vitesse $U$ constante, on obtient l'équation d'advection pure suivante :

$$
\frac{\partial C}{\partial t}+\left(u \frac{\partial C}{\partial x}+v \frac{\partial C}{\partial y}\right)=r
$$

La diffusion (loi de Fick)

La diffusion est le mécanisme de transport de la matière sous l'effet d'un gradient de concentration, depuis les zones concentrées en matière vers les zones moins concentrées. 
La diffusion est aussi le processus pour lequel une substance est déplacée d'un endroit à un autre sous l'action des fluctuations aléatoires [2].

Considérons le système à deux compartiments (la figure II.4), et le flux fluctuant compensé par un anti flux de même grandeur dans la direction opposée.

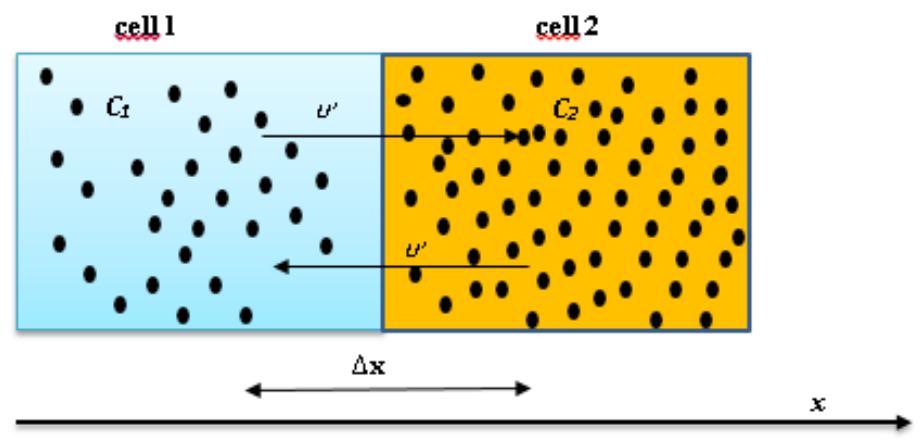

Figure II.4. Schéma expliquant le flux de diffusion

La loi de Fick décrit ce phénomène en énonçant que le flux de matière $J$ est proportionnel au gradient de concentration par l'intermédiaire du coefficient de diffusion $D$.

En faisant le bilan du flux de matière, on obtient :

$$
\begin{aligned}
& \text { flux net de } 1 \text { à } 2=\text { flux de } 1 \text { à } 2-\text { flux de } 2 \text { à } 1 \\
& J=C_{1} u^{\prime}-C_{2} u^{\prime}=-u^{\prime}\left(C_{1}-C_{2}\right)=-u^{\prime} \Delta C=-\left(u^{\prime} \Delta x\right) \frac{\Delta C}{\Delta x}=-D \frac{d C}{d x} \\
& j=-D\left(\frac{d C}{d x}+\frac{d C}{d y}\right)
\end{aligned}
$$

Où $D=$ coefficient de diffusion $\mathrm{m}^{2} / \mathrm{s}$

Dans cette équation, le signe moins traduit le fait que le transfert s'effectue des zones concentrées vers les zones diluées.

- Equation de la diffusion pure

Dans la diffusion pure, on s'intéresse seulement au flux diffusif, et on néglige le flux d'advection.

Soit à écrire le bilan de flux à travers la section $A$ durant le temps $d t$ dans un volume $V$.

Soit $j(x, t) . A$, le flux entrant par $A$ et $j((x+d x), t) . A$, le flux sortant. Partant de l'équation (II.8), on obtient :

$$
\begin{array}{r}
\frac{V d C}{d t}=[j(x, t) \cdot A]-[j(x+\Delta x, t) \cdot A] \\
\Rightarrow \frac{d C}{d t}=-\frac{j(x+\Delta x, t)-q(x, t)}{\Delta x}
\end{array}
$$

En tenant compte du flux interne c'est-à-dire la source, le bilan devient :

$$
\frac{d C}{d t}=-\frac{j(x+\Delta x, t)-q(x, t)}{\Delta x}+r=-\frac{\partial j}{\partial x}+r
$$

En remplaçant l'équation (II.14) dans (II.18), on obtient : 


$$
\frac{\partial C}{\partial t}=-\frac{\partial j}{\partial x}+r=\left(-D \frac{\partial C}{\partial x}\right)+r
$$

En extrapolant l'étude en 2D et pour un coefficient de diffusion $D$ constant, on obtient l'équation de diffusion pure suivante :

$$
\frac{\partial C}{\partial t}-D\left(\frac{\partial^{2} C}{\partial x^{2}}+\frac{\partial^{2} C}{\partial y^{2}}\right)=r
$$

\section{- Equation d'advection-diffusion}

En additionnant le flux advectif au flux diffusif, on obtient le flux total $Q$ suivant :

$$
Q=\text { advection }+ \text { diffusion }=C \cdot \vec{U}-D g r a d C=C u+C v-D \frac{\partial C}{\partial x}+\frac{\partial C}{\partial y}
$$

En suivant la même démarche que dans les cas précédents, on obtient l'équation d'advectiondiffusion suivante :

$$
\frac{\partial C}{\partial t}=-\left(\frac{\partial Q}{\partial x}+\frac{\partial Q}{\partial y}\right)+r=-\frac{\partial(C u)}{\partial x}+\frac{\partial}{\partial x}\left(D \frac{\partial C}{\partial x}\right)-\frac{\partial(C v)}{\partial y}+\frac{\partial}{\partial y}\left(D \frac{\partial C}{\partial x}\right)+r
$$

Si $\boldsymbol{U}, \boldsymbol{V}$ et $\boldsymbol{D}$ sont constants, on a finalement :

$$
\frac{\partial C}{\partial t}+u \frac{\partial C}{\partial x}+v \frac{\partial C}{\partial y}-D\left(\frac{\partial^{2} C}{\partial x^{2}}+\frac{\partial^{2} C}{\partial y^{2}}\right)=r
$$

Où :

- $C:$ concentration d'intérêt en $\left[\mathrm{mg} / \mathrm{m}^{3}\right]$

- $u, v$ : les composantes du vecteur vitesse $U$ suivant les axes $X, Y$ en $[\mathrm{m} / \mathrm{s}]$

- $D$ : coefficient de diffusion en $\left[\mathrm{m}^{2} / \mathrm{s}\right]$

- $r$ : le terme source $\left[\mathrm{mg} / \mathrm{m}^{3} . \mathrm{s}\right]$

\section{Evolution de la concentration du panache de gaz}

Nous avons démontré que le phénomène d'advection diffusion en 2D est régit par l'équation aux dérivées partielles (II.26). Vu que cette équation est non linéaire et qu'elle n'admet pas de solution analytique sans passer par des simplifications, nous recourons à une des méthodes approchées dite méthode des éléments finis développée dans le logiciel Freefem++ [5].

La résolution d'une telle équation en utilisant le logiciel Freefem++ se fait en sept étapes suivantes [6] :

- Définition du domaine d'étude ;

- Discrétisation du domaine d'étude (maillage);

- Définition de l'espace d'approximation ;

- Définition des données du problème et des conditions initiales ;

- Définition du problème variationnel sur l'espace d'approximation ;

- Définition des conditions aux limites ;

- Résolution du problème variationnel ;

- Affichage des résultats.

\subsection{Définition du problème variationnel}

Partant de l'équation (II.27) définissant la formulation forte du problème à résoudre, nous appliquons l'approximation par différences finies au terme de dérivée temporelle, on obtient : 


$$
\frac{C_{i, j}^{k+1}-C_{i, j}^{k}}{d t}+u \frac{\partial C}{\partial x}+v \frac{\partial C}{\partial y}-D\left(\frac{\partial^{2} C}{\partial x^{2}}+\frac{\partial^{2} C}{\partial y^{2}}\right)=r
$$

Ou plus simplement : $\frac{C_{i, j}^{k+1}-C_{i, j}^{k}}{d t}+U \cdot \nabla C_{i, j}^{k}-D \cdot \Delta C_{i, j}^{k}=\mathrm{r}$

Avec $U=u \cdot \vec{\imath}+v \cdot \vec{\jmath}$

En multipliant l'équation (II.29) par la fonction test $C_{h}$ et en appliquant le théorème de Green Ostrogasky, on obtient :

$$
\begin{gathered}
C h \cdot\left[\frac{C_{i, j}^{k+1}-C_{i, j}^{k}}{d t}\right]+C h \cdot U \cdot \nabla C_{i, j}^{k}+D \cdot \nabla C_{i, j}^{k} \cdot \nabla C h=r . C h \\
\text { Ch. } C_{i, j}^{k+1}-C h . C_{i, j}^{k}+d t \cdot C h \cdot U \cdot \nabla C_{i, j}^{k}+d t \cdot D \cdot \nabla C_{i, j}^{k} \cdot \nabla C h=r . C h . d t
\end{gathered}
$$

En intégrant 1'équation (II.29) membre en membre, on obtient :

$$
\begin{gathered}
\iint_{\Omega} C h \cdot C_{i, j}^{k+1} \cdot d x \cdot d y-\iint_{\Omega} C h \cdot C_{i, j}^{k} \cdot d x \cdot d y+\iint_{\Omega} d t \cdot C h \cdot U \cdot \nabla C_{i, j}^{k} \cdot d x \cdot d y+\iint_{\Omega} d t \cdot D \cdot \nabla C_{i, j}^{k} \cdot \nabla C h \cdot d x \cdot d y \\
=\iint_{\Omega} r \cdot C h \cdot d t \cdot d x \cdot d y \quad(I I \cdot 30)
\end{gathered}
$$

L'équation (II.30) obtenue constitue la formulation variationnelle (faible) du modèle mathématique appliqué à notre système.

\subsection{Définitions des conditions initiales et aux limites}

La notion de conditions aux limites se perçoit ici comme les conditions sur une frontière délimitant la zone d'observation et non comme celles d'une frontière physique entrainant un changement brutal de milieu. Numériquement, nous allons considérer un domaine discret, fermé, sur lequel nous effectuerons les calculs de concentration. Ce domaine sera délimité par une frontière virtuelle discrète $\partial \Omega$, d'épaisseur une maille autour du domaine $\Omega$. L'ensemble constitue donc un domaine $\partial \Omega+\Omega$ fermé. N'ayant en fait aucune connaissance sur la concentration du contour $\partial \Omega$, nous la fixerons à zéro.

En considérant le milieu sain avant l'émanation du polluant, nous fixons donc la concentration initiale $C_{1}$ dans le domaine égale à zéro.

\subsection{Données du problème}

Les données du problème sont :

- Longueur du domaine suivant l'axe x : 15 unités de longueur ;

- Largeur du domaine suivant l'axe y : 15 unités de longueur ;

- La concentration du polluant dégagé depuis la source étant fixé à $10 \mathrm{mg} / \mathrm{m}^{3}$;

- Le coefficient de diffusion $D: 1.24 \mathrm{~m}^{2} / \mathrm{s}$;

- Coordonnées de la source : $x=3,5$ et $y=3$;

- Vitesse du vent : $u=v=2,7 \mathrm{~m} / \mathrm{s}$;

- Présence de quelques obstacles définis par leurs conditions aux limites. 


\section{Résultats et interprétation}

Pour un temps de simulation de 5 unités, pour un pas de 0.5 et pour une simulation en tenant compte de la sensibilité du maillage, on obtient les résultats ci-dessous :

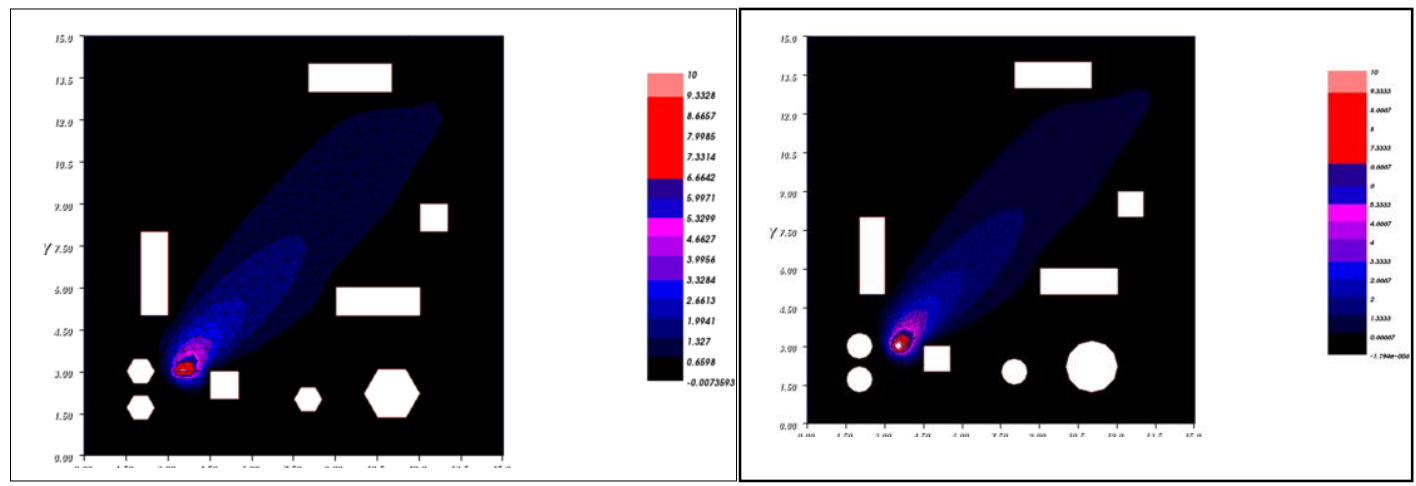

(a)

(b)

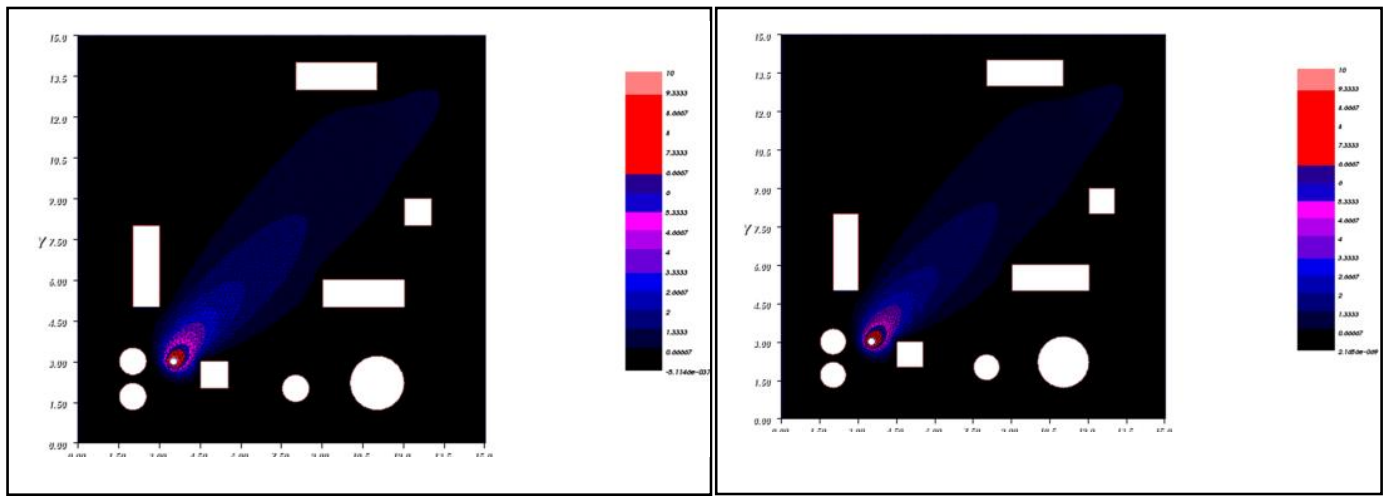

$(c)$

$(d)$

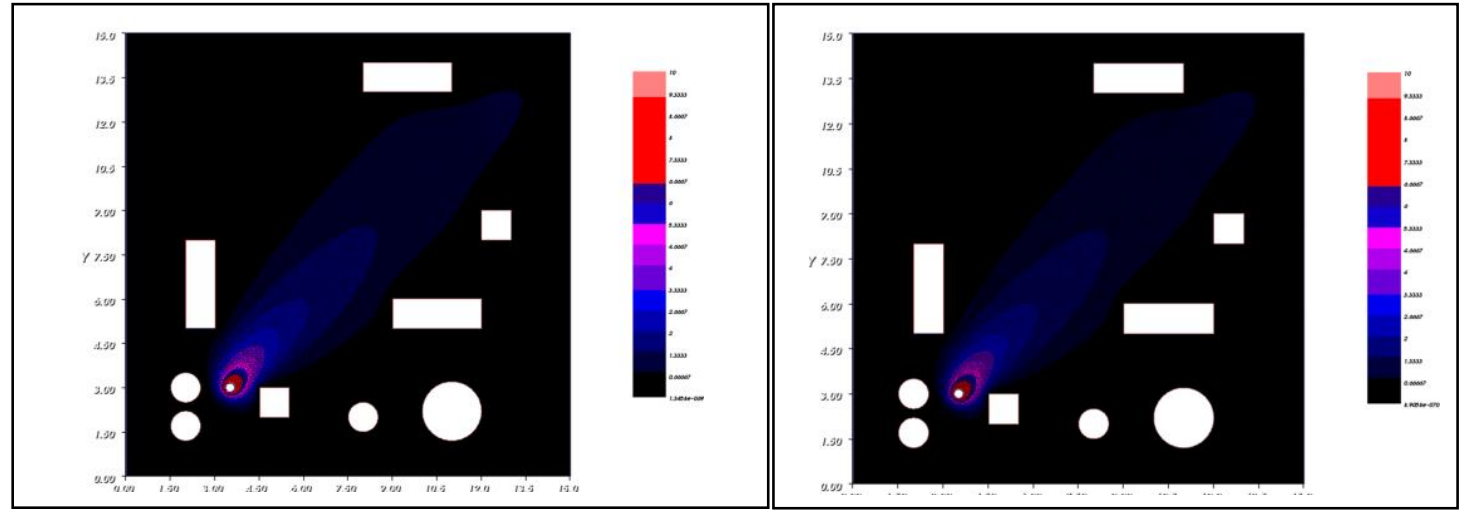

$(e)$

(d)

Figure II.5. Simulation du flux de gaz pour six niveaux de résolution de maillage

Nous constatons une dégradation de la concentration du gaz durant sa propagation (cf. légende). Le résultat montre aussi que les zones non impactées donc hors de danger sont presque en noir. Nous voyons aussi que la propagation du gaz se fait suivant direction et le sens d'écoulement du vent. Les zones environnant la source d'émanation constituent de zones de forte concentration. Et l'étude de la sensibilité de maillage montre que la configuration du maillage dont le résultat présenté 
à la figure II.5c offre une précision optimale (écart de résultat inférieur à 5\%) pour un temps d'exécution 9,461 secondes.

\section{Conclusion et perspective}

La résolution du problème d'advection diffusion montre que l'étendue de la zone impactée est directement proportionnelle à la vitesse de propagation (du vent) et au coefficient de diffusion. Le niveau de concentration du polluant n'affecte que très minimalement cette étendu. Nous suggérons comme suite à ce travail, une étude d'estimation de coordonnées d'une source en cas d'une émanation accidentelle.

\section{Références}

[1] Bruno Sportisse, Modélisation et simulation de la pollution atmosphérique, Université Pierre et Marie Curie, 2007

[2] Victor Winiarek, Dispersion atmosphérique et modélisation inverse pour la reconstruction de sources accidentelles de polluants, Université Paris-Est, 2014

[3] Medaouar WALID, Simulation numérique du phénomène d'advection et de diffusion de la concentration d'un polluant dans un écoulement permanent bidimensionnel par la méthode des volumes finis, Université Hassiba Benbouali de Chlef, 2012

[4] Robert Perrin, Jean Pierre Scharff, Chimie industrielle. Dunod $2^{\text {ème }}$ Edition, Paris 2002

[5] Daniel Choi, Méthode des éléments-finis par l'exemple, Université de Caen, 2010

[6] Fréderic Hecht, FreeFem++, 2009 